\title{
La investigación educativa, empleo de tecnologías digitales en la formación permanente
}

\section{Educational research, use of digital technologies in lifelong learning}

\author{
PhD Maricela Pérez Medina ${ }^{1}$ \\ maricelap@uclv.cu \\ www.orcid. 0000-0002-5593-5132
}

Recibido: 1/4//2020; Aceptado: 1/6/2020

\begin{abstract}
RESUMEN
El objetivo de este artículo está encaminado al análisis de los retos y tendencias de la investigación educativa acerca del aprendizaje empleando la tecnología digital, al aprobarse el Plan E de la Licenciatura en Educación, Educación Laboral. Muchas investigaciones han versado sobre el uso de las tecnologías digitales para la formación y el aprendizaje de las asignaturas en la Disciplina Procesos Constructivos y un problema se ha venido consolidando en el ámbito de la investigación multidisciplinar integrando las didácticas del resto de las demás disciplinas con ejercicios integradores; pero en los resultados obtenidos en las validaciones de las asignaturas se muestran algunas carencias en ello, ya que no se da la respuesta necesaria que se exige en el Modelo del Profesional. Será necesario diseñar ayudas tecno-pedagógicas adecuadas que favorecerán el aprendizaje de los estudiantes de la carrera. La autora considera que es el momento de reflexionar sobre la utilización de nuevos métodos y herramientas de trabajo que puedan mejorar la calidad y los resultados de las investigaciones desde lo curricular y a su vez el mejoramiento del proceso de enseñanza aprendizaje de la Disciplina Procesos Constructivos. Para ello se han tenido en cuenta como objetivo crear un repositorio digital para la difusión del conocimiento, teniendo en cuenta la relación inter y multidisciplinar para que los estudiantes se auto gestionen su aprendizaje y ello se ha concebido desde la investigación científica en las clases de Taller de Materiales Variados y Confecciones Textiles, con énfasis en el Tejido a Crochet.
\end{abstract}

Palabras clave: repositorio, creatividad, investigación

\section{ABSTRACT}

This article aims at analyzing the challenges and trends in educational research in learning through the use of digital technology, given the approval of Plan E for the Degree in Education, Labor Education. This research is focused on the use of digital technologies for the training and learning of the subjects in the Discipline of construction processes. A problem has been consolidated in the field of multidisciplinary research incorporating the didactic of the other disciplines with integrative exercises, but the results obtained in the validations of the subjects show some deficiencies regarding this, since the necessary response required in the

\footnotetext{
${ }^{1}$ Universidad Central "Marta Abreu" de Las Villas, Santa Clara, Villa Clara, Cuba
} 


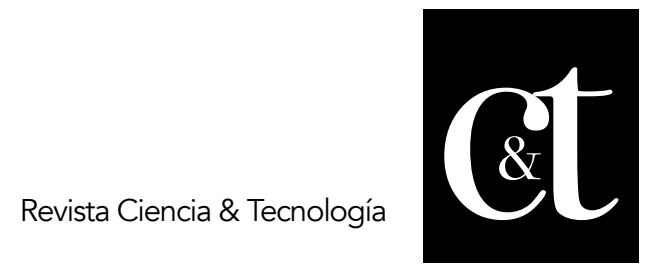

No. 27, 31 de julio de 2020

ISSN impreso: 1390 - 6321

ISSN online: 2661 - 6734

Professional Model is not provided, so that appropriate techno-pedagogical aids are designed that will favor the learning of the students of the career. The author considers that it is time to make a reflection about using new methods and teaching tools that can improve the quality and results of research from the curricular point of view and at the same time improve the process of teaching and learning of the Construction Processes Discipline. For this purpose, it has been taken into consideration the objective of creating a digital repository for the dissemination of knowledge, taking into account the inter- and multidisciplinary relationship so that students can manage their own learning, and this has been conceived from scientific research in the classes of Workshop of Varied Materials and Textile Confection, with emphasis on the Weaving to Crochet.

\section{Keywords: repository, creativity, research}

\section{Introducción}

El objetivo de este artículo está encaminado al análisis de los retos y tendencias de la investigación educativa acerca del aprendizaje empleando la tecnología digital y además de las tendencias y retos futuros de la misma en la carrera de la Licenciatura en Educación, Especialidad Educación Laboral Plan E, que tiene sus antecedentes en el Plan D. En los últimos años las investigaciones se dirigían más a la resolución de problemas de las asignaturas Didáctica de la Informática que a la de Educación Laboral; no obstante, los resultados obtenidos muestran algunas limitaciones en el aprendizaje de los contenidos teóricos y prácticos en la Disciplina Procesos Constructivos.

Sin embargo, cuando la autora realiza al análisis de constatación en la práctica pedagógica, se observa que han existido limitaciones en lograr el autoaprendizaje de los estudiantes debido a la carencia de trabajos que proporcionen recursos didácticos informáticos y de otros que contribuyan a lograr la inter y multidisciplinariedad de los contenidos de las asignaturas que conforman las disciplinas de la especialidad que inciden en el aprendizaje, auto aprendizaje y formación de habilidades específicas en los estudiantes. Tampoco hay propuestas de medios de enseñanza digitales para emplearlos en la docencia, de manera que permitan el desarrollo investigativo en ellos desde las propias asignaturas que reciben en su plan de estudio y que culminan con Trabajo de Curso como examen final.

Estudios realizados sobre el empleo de las TICs para el aprendizaje de los estudiantes ha formado parte de un ámbito de estudio amplio y multidisciplinar, combinando miradas e intereses muy diversos. Sin embargo, disímiles investigaciones intervienen en un mismo problema: no facilitan respuestas suficientes que permitan analizar, planificar, diseñar y ejecutar prestaciones tecno-pedagógicas adecuadas para favorecer el aprendizaje eficiente y desarrollo de habilidades de los estudiantes. En ocasiones, se aprecia una investigación superficial y que no se profundiza en el empleo de nuevos métodos científicos para penetrar en el problema declarado, intervenir y así poder transformarlo y darle solución.

Durante todos estos años de empleo de las TICs en el proceso de enseñanza aprendizaje, se ha demostrado, en la constatación en la práctica educativa, que por el mero hecho de su empleo se logra el mejoramiento en el aprendizaje de los estudiantes, siendo muy frecuente encontrar trabajos investigativos empleando las TICs en las diferentes educaciones sustentados sobre un punto de vista muy 


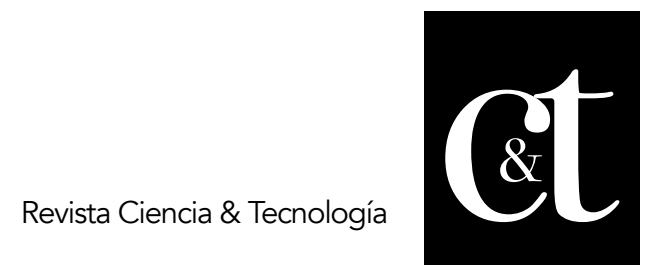

No. 27, 31 de julio de 2020

ISSN impreso: 1390 - 6321

ISSN online: 2661 - 6734

inexperto que considera que la tecnología transforma las prácticas educativas por sí solas. Pero, también están los opuestos, quienes creen que con su empleo como herramienta se facilitarán ciertas prácticas. Pero lo importante es la metodología que se emplee. En los tiempos actuales, es el empleo de las tecnologías con diseños educativos pedagógicos empleando métodos y procedimientos adecuados los que permiten partiendo de un diagnóstico del grupo con el que se interactúa, trasformando la realidad educativa detectada como problema.

El contexto mundial complejo en el que se adentra la sociedad cubana requiere que todo profesor posea la preparación necesaria para utilizar la tecnología digital con eficacia desde la investigación, buscando alternativas de solución a los problemas declarados en su asignatura a partir del conocimiento de las características del o de los grupos con que trabajará o trabaja. De esta manera incide en su formación permanente y su desempeño profesional, contribuyendo de esta forma a la formación integral de los estudiantes con las habilidades profesionales necesarias en un mundo globalizado en información y conocimiento. Una de las asignaturas de formación básica y que contribuye a ello es la disciplina de Formación Laboral Investigativa (FLI), que los forma y prepara para enfrentarse al mundo laboral de forma responsable.

\section{Desarrollo}

La educación en el contexto actual de perfeccionamiento que lleva a cabo el Ministerio de Educación en todas las enseñanzas del país, tiene la tendencia de irse desarrollando como un sistema abierto y permanente que exige la innovación de los profesores para favorecer: el estudio autónomo de sus estudiantes, la autogestión de su aprendizaje, la investigación constante, el trabajo en equipo, el desenvolvimiento de procesos interactivos de comunicación y construcción del conocimiento, mediados por la acción del diálogo entre profesores y estudiantes, todo ello basado en el desarrollo de competencias para su formación laboral, profesional y para el empleo. Por tales razones, es imprescindible plantear intervenciones desde la investigación que promuevan la adquisición de las habilidades que los estudiantes universitarios precisan para aprender de forma independiente, de modo que se les debe enseñar cómo hacerlo e intensificar las habilidades con las que ya cuentan y desarrollan desde el primer año con el trabajo extracurricular. La carrera de Licenciatura en Educación, Educación Laboral Plan E en su perfeccionamiento concibió en sus programas de asignaturas y disciplinas esta concepción pedagógica para el aprendizaje de sus estudiantes, concretado en la Disciplina FLI.

Esta disciplina se presenta en el plan E con una nueva concepción, en la que ocupan un papel significativo el estudio de los contenidos de la metodología de la investigación y la Didáctica particular de la Educación Laboral, que posibilitan la integración de los contenidos adquiridos en la formación básica, formación básica especializada y formación básica de la profesión, mediante la concreción de las situaciones profesionales del proceso pedagógico en general y el de enseñanzaaprendizaje de la asignatura Educación Laboral en las instituciones educativas y, la solución a problemas de la profesión mediante el método científico, así como la preparación para el ejercicio de culminación de estudios. Esta concreción se logra mediante la integración de los componentes académico, laboral e investigativo, y la extensión universitaria.

El componente laboral desde sus inicios facilitó el adecuado vínculo entre las actividades docentes, las prácticas pedagógicas y el trabajo científico-estudiantil, 


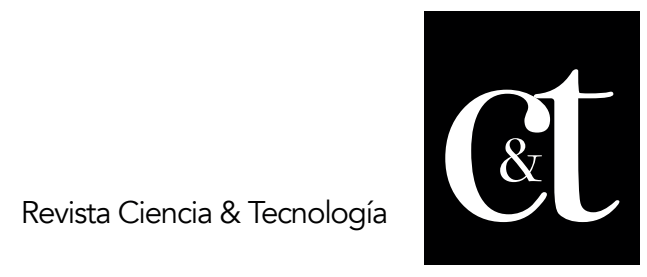

No. 27, 31 de julio de 2020

ISSN impreso: 1390 - 6321

ISSN online: 2661 - 6734

organizándose en los diferentes planes de estudios, prácticas sistemáticas, concentradas y a tiempo completo en las instituciones educativas, donde los estudiantes de la carrera a partir del análisis del banco de problemas seleccionan el tema de su investigación atendiendo a las carencias de su grupo de trabajo y lo desarrolla desde la universidad para contribuir a solucionar el mismo.

Para dar solución al problema declarado a partir de una correcta organización, planeación, ejecución y evaluación de la propuesta pedagógica empleando las posibilidades que brindan los medios tecnológicos y la investigación, es esencial tenerse en cuenta la formación integral y laboral de los estudiantes. El objetivo esencial de la formación laboral en la carrera es la aplicación de los conceptos de cómo aprender a aprender, aprender a ser, aprender a hacer, cómo desarrollar sus capacidades y habilidades para continuar aprendiendo haciendo con sus manos desde el desempeño profesional futuro.

Por la necesidad de este tipo de aprendizaje declarado anteriormente, en el proceso de enseñanza-aprendizaje de la Disciplina FLI, en la formación permanente de los estudiantes de Educación Laboral, se une la problemática de que no es suficiente el tiempo destinado al desarrollo de habilidades manuales durante las clases prácticas de algunas asignaturas del Modelo del Profesional como es el caso de la asignatura Taller de Materiales Variados y Confecciones Textiles que forma parte de la disciplina Procesos Constructivos. Por los contenidos incorporados al programa de la misma, y teniendo en cuenta que se disminuye en años la formación de pregrado, los profesores deben buscar otros espacios educativos en los cuales el estudiante pueda realizar acciones dirigidas al desarrollo teórico-práctico, en el cual puedan participar de forma individual o colectiva, donde el profesor constate la correcta representación de artículos de utilidad social, el desarrollo de la creatividad, iniciativa, interpretación de símbolos y gráficos, independencia, entre otros, reforzando así los contenidos teóricos recibidos en el taller de manualidades y su relación interdisciplinaria como en (Borroto, 2016).

Que los estudiantes sean capaces de explicar los pasos seguidos para la representación del artículo y los métodos empleados en la solución de la problemática planteada en clases, escuela y la sociedad, esto solo se logra desde la investigación personalizada de ellos, desde los contenido de las asignaturas y desde su formación académica donde se preparan para utilizar las tecnologías de la información y las comunicaciones (TIC) con eficacia, eficiencia y ética, tanto en su formación, como en su desempeño profesional, al tributar al modelo del profesional con los contenidos necesarios para su utilización como medio de enseñanza y herramienta de trabajo en el proceso de enseñanza-aprendizaje de la asignatura. En el artículo se destacan las ventajas que brinda el empleo de las TICs en la investigación para el desarrollo de habilidades manuales e intelectuales de los estudiantes, incidiendo en el aprendizaje para resolver los problemas propuestos, sin ignorar los aspectos cognitivos, pero sí relacionándolos con la creación de nuevos modelos y modalidades educativas.

Cada vez se hace imprescindible la investigación en educación con el uso de medios digitales, ya que es un proceso para renovar y transformar los ambientes de enseñanza aprendizaje, logrando calidad en la educación, es decir, que responda a las necesidades de los estudiantes según sus contextos educativos. La investigación es factor necesario en los procesos educativos, permite transformaciones favorables: la investigación proporciona nuevas formas de comprensión sobre la dinámica de los 


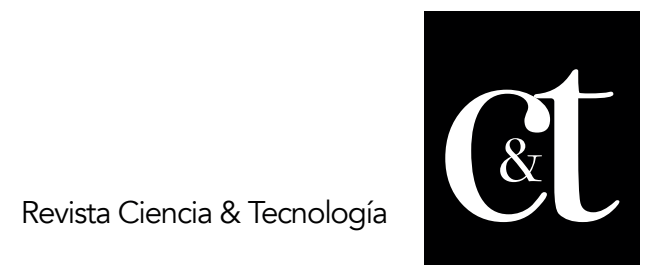

No. 27, 31 de julio de 2020

ISSN impreso: 1390 - 6321

ISSN online: 2661 - 6734

procesos de enseñanza y aprendizaje tanto para el profesor, como para el estudiante (De Pablos, 2013).

Esta peculiaridad de la investigación desde lo docente, permite desarrollar en los estudiantes un espíritu crítico, constructivo y renovador de la realidad. Fomenta la búsqueda del conocimiento, el desarrollo del pensamiento científico y de las habilidades científico investigativas, que es la base para encontrar soluciones a los problemas profesionales. Un elemento no menos importante, es su contribución a la elevación de los niveles de compromiso, motivación e identidad profesional, en tanto les permite vivenciar la utilidad de su profesión y de su propio trabajo pedagógico en los centros de práctica laboral.

Por ello, en la actualidad se hace imprescindible que las clases de la Especialidad Educación Laboral contribuyan cada día al desarrollo de una cultura general integral en los profesionales de la carrera a partir del dominio de recursos, conceptos y procedimientos informáticos vinculados a la investigación. No se trata de saber si se aprende mejor, sino qué se aprende y lo que realmente interesa es comprender el cómo. En otras palabras, se necesita entender cómo diseñar entornos virtuales y situaciones educativas que puedan mejorar el aprendizaje desde la investigación. La investigación y la innovación se han convertido en una pieza clave en el logro de una educación que impulse el desarrollo humano, económico, político y social, y se convierta en un instrumento real de mejora del bienestar individual y colectivo.

Según lo abordado y tenido en cuenta en el Modelo del Profesional de la Carrera de Licenciatura en Educación, especialidad Educación Laboral, la formación del profesional debe ser, innovadora y creativa, poseedora de una cultura científica y pedagógica y con un alto compromiso social el de esta carrera que es la encargada de dirigir el Sistema de Acciones Laborales en la Secundaria Básica, enseñanza para la cual se forman los estudiantes, aunque pueden tener su campo de acción en la Enseñanza Técnica Profesional y en las Escuelas de Oficios.

Innovadora implica el uso de medios digitales. De ahí que sea necesario lograr la calidad de los egresados, reconociendo tanto el nivel de preparación teórica adquirida en esta y otras asignaturas que tributan a ella, como la capacidad de enfrentar, con independencia, responsabilidad, originalidad, ética y creatividad, las tareas profesionales a su cargo y su participación protagónica y activa de su propio proceso formativo.

Según Álvarez de Zayas (1997), el proceso educativo abarca la educación formalizada que transcurre en las instituciones educativas donde se reflejan las aspiraciones, cultura, historia, costumbres, así como conflictos y demandas de la sociedad; y la educación no formalizada que se produce a nivel de toda la sociedad a través de los medios masivos de comunicación, los medios informáticos, las instituciones culturales y deportivas, los centros de entretenimiento; los grupos y asociaciones, todos los cuales actúan con sus contenidos, con sus mensajes, con su estilo, sobre los miembros de la comunidad, formándolos positiva o negativamente (ver Álvarez de Zayas, Hacia un currículum integral y diferenciado. 1997:11-12).

El contenido de la investigación científica deviene contenido del aprendizaje en programas didácticamente organizados, que propician el enfoque interdisciplinario como fundamento epistemológico. De la lógica de la ciencia y de su papel en la formación del profesional de la educación resulta la secuencia de disciplinas y 


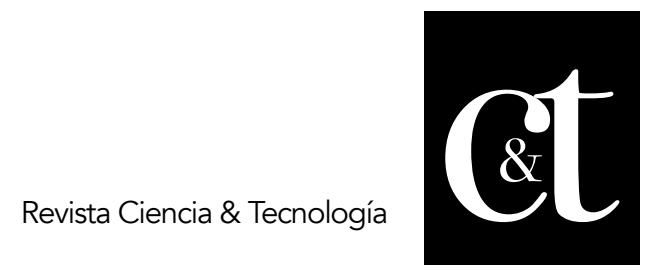

No. 27, 31 de julio de 2020

ISSN impreso: 1390 - 6321

ISSN online: 2661 - 6734

actividades. En este aspecto, el enfoque interdisciplinario le aporta flexibilidad y un carácter abierto al proceso de enseñanza aprendizaje. Es por ello, que el profesional de la educación desde la investigación científica debe estar preparado para la creación de proyectos educativos, cuya mirada más global permita la creación de acciones que rebasen el marco disciplinar, para orientar metas más integradoras que requieren de la implicación de todo el colectivo pedagógico. Todo esto con uso de las TIC.

El profesor debe lograr las siguientes habilidades en sus estudiantes:

Resolver problemas correspondientes a los componentes de su formación profesional académica, laboral e investigativo con el empleo de las TIC.

Fundamentar la utilización de la computadora como medio de enseñanza y como recurso técnico en su investigación.

Evaluar desde el punto de vista pedagógico los recursos vinculados con el proceso de enseñanza aprendizaje de la Educación Laboral disponibles en las redes informáticas.

Modelar actividades docentes para el proceso de enseñanza-aprendizaje de Educación Laboral con el apoyo de las TIC.

Resolver problemas vinculados a tareas del diseño de artículos de utilidad social, así como de otros medios necesarios para su labor profesional. (Pérez Medina, 2019).

\section{Resultados}

Un ejemplo de resultado de la investigación en la formación de pregrado donde se emplea el recurso informático es el repositorio digital para el contenido del Tejido a Crochet, que da solución a las carencias que hoy se presenta en este tema tanto en bibliografía impresa, como digital en la asignatura Educación Laboral en el 9no grado de Secundaria Básica y en la Licenciatura en Educación con el mismo nombre, donde no existe un texto o material impreso o digital que aborde propuestas de artículos a realizar en la asignatura, orientaciones de cómo hacerlo, qué material y e instrumentos emplear, cómo interpretar la simbología en este tema para realizar las aplicaciones, cómo desarrollar la creatividad partiendo de un artículo ya elaborado, entre otros aspectos educativos, siendo contenidos que se incorporaron en el perfeccionamiento de la asignatura en el MINED y MES (cfr. Paredes, 2013).

Para su confección se tomaron como base fundamental elementos teóricos y gráficos de sitios de internet a partir de la búsqueda derivada de la investigación en el tema y experiencias de la autora. Se tuvo en cuenta que el enfoque del contenido del repositorio se ajustara a los diferentes niveles de aprendizaje de los estudiantes del grado y año, así como con los recursos con los que se cuenta en la actualidad en las escuelas, aunque puede construirse por los profesores y los mismos estudiantes la agujeta de tejer en el Taller de Carpintería a la medida que se desee. El objetivo que se persigue es el de contar con un repositorio digital que recoja los contenidos básicos de la unidad en ambas enseñanzas y que los estudiantes puedan realizar actividades prácticas, para el estudio individual, desarrollar la creatividad y auto prepararse desde la investigación de sus temas, esto permitirá profundizar en ellos y buscar variedad en diseños creativos y originales pudiendo transformarlos según los recursos con que cuenten en sus centros.

Los repositorios son considerados "un sistema de gestión de contenidos, que administra la producción científica en formato digital. Utilizan estándares abiertos para garantizar que sus contenidos sean accesibles y puedan ser buscados y recuperados para su uso posterior. Son un medio de publicación científica. Ofrece 


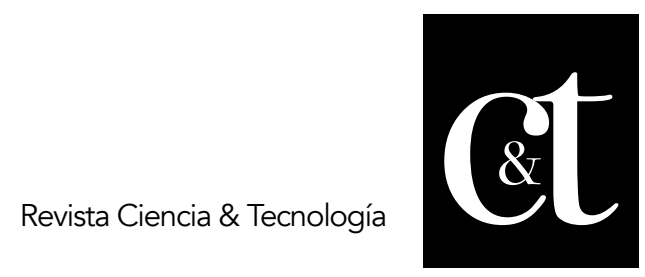

No. 27, 31 de julio de 2020

ISSN impreso: 1390 - 6321

ISSN online: 2661 - 6734

otros servicios complementarios" (Ecured. Repositorio.html. Consultado el 18 de marzo 2020). Un repositorio contiene y permite importar, identificar, almacenar, preservar, recuperar y exportar un conjunto de objetos digitales, desde un portal web. El objetivo de los repositorios es: recopilar y organizar los documentos digitales de carácter científico, docente e institucional producidos por determinada entidad para el apoyo a la investigación, docencia y aprendizaje. De esta manera se mejora la visibilidad de la producción científica y académica de una universidad o centro de investigación, permitiendo el acceso abierto a sus contenidos y garantizando la preservación y conservación de dicha producción, por ello desde la investigación científica se utilizó como resultado de investigación para ser utilizado como medio de enseñanza en clases, específicamente en la Unidad 2 de 9no grado.

En artículo presentado por Polanco-Cortés sobre repositorios digitales definición y pautas para su creación en Universidad de Costa Rica señala que: Un repositorio digital es un medio para gestionar, almacenar, preservar, difundir y facilitar el acceso a los objetos digitales que alberga", los autores consideran que este resultado científico responde al de tipo disciplinar o temático, y da solución al problema científico de la Secundaria Básica, el repositorio se organizó atendiendo a los siguientes temas:

La historia del ganchillo.

Todos los diferentes tipos de herramientas y materiales necesarios para tener éxito.

Utilización de la técnica de hilado correctamente.

Aprender el lenguaje del ganchillo.

Puntos básicos para empezar.

Aplicaciones con su paso a paso.

Videos Educativos.

Descubrir las innovaciones del crochet.

Los consejos secretos de expertas sobre qué técnicas utilizar.

Diferentes técnicas del uso del ganchillo.

Ayuda en la elección de la cinta de medir según las necesidades del ganchillo. Cómo utilizar un contador de la fila correctamente.

Investigar de cómo convertir las cadenas.

Vocabulario Técnico.

Sección de curiosidades.

Actualmente, la mayoría de las universidades disponen de repositorios institucionales como resultado científico como recurso educativo, de esta forma permiten distribuir la creación científica, incluyendo informes científicos y técnicos, informes de trabajos de Diplomas, artículos y datos. Tener un repositorio supone una ventaja profesional para la propia formación ya que le proporciona un valor añadido al incrementar la visibilidad y el impacto de sus investigadores en el entorno educativo.

\section{Conclusiones}

La investigación sobre el uso de las TICs en la educación constituye un ámbito consolidado de estudio que todavía tiene algunas limitaciones, es necesario superar una aproximación simplificadora e incorporar a los procesos acciones que orienten la investigación y permitan comprender la interacción dinámica entre el aprendizaje y las prestaciones tecnológicas. Esta aproximación obliga a avanzar en la utilización de nuevos métodos e instrumentos de investigación. 


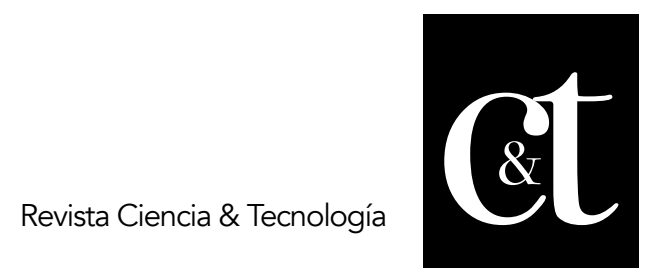

No. 27, 31 de julio de 2020

ISSN impreso: 1390 - 6321

ISSN online: 2661 - 6734

La aplicación de las tecnologías en las clases con el empleo de repositorios y las redes de la información posibilita que los resultados de la investigación puedan estar a disposición del resto de la comunidad científica de manera universal e inmediata. El acceso abierto a los resultados de la investigación modifica la propia investigación que necesariamente pasa a ser una producción grupal y colaborativa.

\section{Referencias bibliográficas}

Álvarez de Zayas R.M. (1997). Hacia un currículo integral y contextualizado. La Habana: Editorial Pueblo y Educación; 1997. p. 106.

Borroto, M. (2016). Taller docente I. Editorial Pueblo y Educación. La Habana.

Ecured (2020). Repositorio. Ecured. Repositorio.html. (Consultado el 18 de marzo 2020).

De Pablos, J. (2013). «La formación e investigación en el campo de la tecnología educativa». Revista Fuentes, 13, pp. 9-16

MES (2016). Plan de estudio de la Licenciatura en Educación Especialidad Educación Laboral Indicaciones metodológicas y de organización de la carrera. La Habana.

Padrón P. E. y Pérez M. (2013). Taller I Papel, Cartulina, Cartón y Fibras Textiles. Editorial Pueblo y Educación. La Habana.

Pérez, M. (2019). Programa de la asignatura Informática Básica aplicada al Dibujo 2do año CD. Especialidad Educación Laboral Plan E. Santa Clara. Villa Clara. MES.

Paredes, J. (2013). «Políticas educativas públicas sobre tic en España. Tres décadas donde los docentes universitarios influyeron en el cambio educativo». Revista Fuentes, 13, pp. 45-78.

Polanco, C, J. (2014). Repositorios digitales definición y pautas para su creacion.pdf. Artículo consultado. Universidad de Costa Rica. 